\title{
Accuracy of Bone Measurements in the Vicinity of Titanium Implants in CBCT Data Sets: A Comparison of Radiological and Histological Findings in Minipigs
}

\author{
Alexander Gröbe, ${ }^{1}$ Jan Semmusch,, Maximilian Schöllchen, ${ }^{1}$ Henning Hanken, \\ Michael Hahn, ${ }^{2}$ Wolfgang Eichhorn, ${ }^{1}$ Gerhard Schön, ${ }^{3}$ Ole Jung, ${ }^{1}$ Jamal M. Stein, ${ }^{4}$ \\ Aline Reitmeier, ${ }^{5}$ Max Heiland, ${ }^{6}$ Ralf Smeets, ${ }^{1}$ and Clarissa Precht ${ }^{1}$ \\ ${ }^{1}$ Department of Oral and Maxillofacial Surgery, University Medical Center Hamburg-Eppendorf, Hamburg, Germany \\ ${ }^{2}$ Department of Osteology and Biomechanics, University Medical Center Hamburg-Eppendorf, Lottestraße 59, \\ 22529 Hamburg, Germany \\ ${ }^{3}$ Department of Medical Biometry and Epidemiology, University Medical Center Hamburg-Eppendorf, Hamburg, Germany \\ ${ }^{4}$ Department of Operative Dentistry, Periodontology and Preventive Dentistry, RWTH Aachen University Hospital, Aachen, Germany \\ ${ }^{5}$ Department of Laboratory Animal Science, University Medical Center Hamburg-Eppendorf, Hamburg, Germany \\ ${ }^{6}$ Charité-Universitätsmedizin Berlin, Corporate Member of Freie Universität Berlin, Humboldt-Universität zu Berlin, \\ and Berlin Institute of Health, Department of Oral and Maxillofacial Surgery, Berlin, Germany \\ Correspondence should be addressed to Clarissa Precht; cprecht@uke.de
}

Received 30 March 2017; Revised 30 May 2017; Accepted 12 June 2017; Published 17 July 2017

Academic Editor: Eiichi Honda

Copyright (C) 2017 Alexander Gröbe et al. This is an open access article distributed under the Creative Commons Attribution License, which permits unrestricted use, distribution, and reproduction in any medium, provided the original work is properly cited.

\begin{abstract}
Purpose. The aim of this animal study was the determination of accuracy of bone measurements in CBCT (cone-beam computed tomography) in close proximity to titanium implants. Material and Methods. Titanium implants were inserted in eight Göttingen minipigs. 60 implants were evaluated histologically in ground section specimen and radiologically in CBCT in regard to thickness of the buccal bone. With random intercept models, the difference of histologic measurements and CBCT measurements of bone thickness was calculated. Results. The mean histological thickness of the buccal bone was $5.09 \mathrm{~mm}$ (CI $4.11-6.08 \mathrm{~mm}$ ). The four raters measured slightly less bone in CBCT than it was found in histology. The random effect was not significant ( $p$ value 1.000). Therefore, the Intraclass Correlation Coefficient (ICC) was 98.65\% (CI 100.00-96.99\%). Conclusion. CBCT is an accurate technique to measure even thin bone structures in the vicinity of titanium implants.
\end{abstract}

\section{Introduction}

Cone-beam computed tomography (CBCT) was introduced in medical imaging in 1998 by Mozzo et al. [1]. While the authors intended this technique as a computed tomography device in dental imaging, СBCT has extended its indications ever since. In recent guidelines from 2013, the indications are formulated such as assessment of pathologies in dental root structures, preoperative trauma diagnostics, imaging of intraosseous lesions, and implantologic planning [2]. Apart from the head and neck region, there are possibilities of imaging of the female breast or picturing osseous lesions of the wrist $[3,4]$. Compared to the multislice computed tomography (MSCT), there is a lack of soft tissue discrimination, but its resolution of high contrast structures provides diagnostic value, which can even exceed MSCT [5]. Even in detection of osseous invasion of oral squamous cell carcinoma, CBCT could be valuable [6]. Good image quality and a relatively low radiation exposure compared to MSCT are the benefits of this technique [7-12].

For a correct interpretation of the three-dimensional dimensions of anatomical and pathological structures in the head and neck region, an accurate acquisition and visualization are required to perform sufficient measurements. 

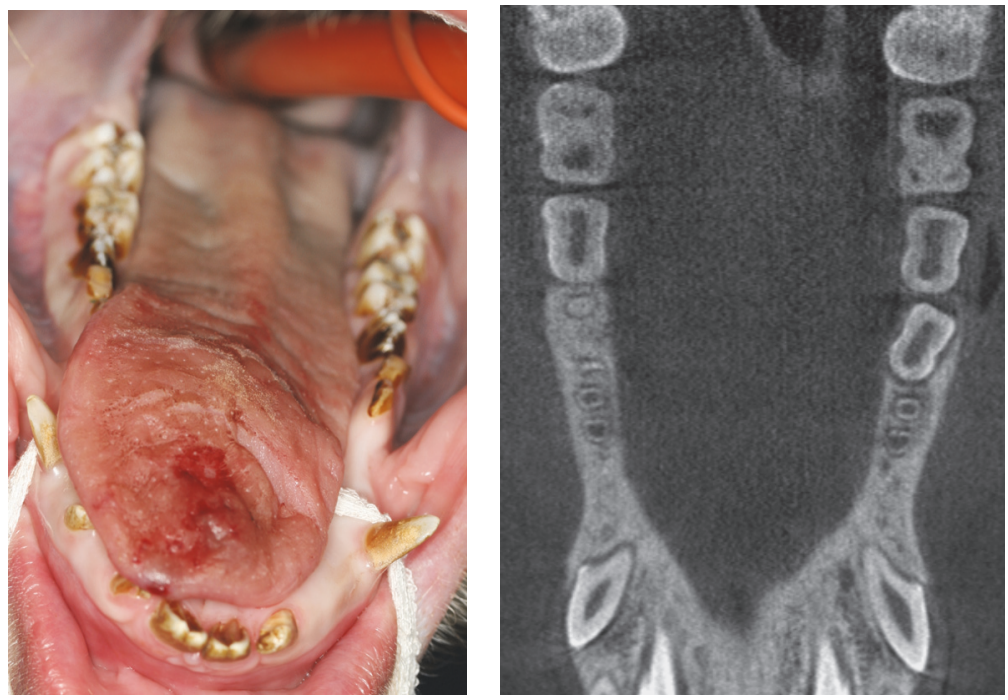

FIGURE 1: Clinical assessment and CBCT before surgery.

In this context, Guijarro-Martínez and Swennen showed that CBCT is suitable for measurements and analysis of the upper airway [13]. Friedrich et al. found CBCT suitable for the determination of orbital volumes, while other authors evaluated its imaging value in measurement of Schneiderian membrane thickness, visualization of apical periodontitis, and reliability of volume data sets using virtual models [1417].

Even though artifacts can lower image quality to a certain extent, CBCT is generally considered to provide sufficient data for measurements of bone volume even in the vicinity of titanium dental implants [18-21]. The purpose of the present study was to evaluate this accuracy by using an animal model and comparing bone measurements of a thin buccal lamella of CBCT datasets with corresponding histological sections.

\section{Materials and Methods}

2.1. Animals. The laboratory animals were eight healthy, fullgrown minipigs (Göttingen Minipig ${ }^{\circledR}$ ) [22], aged 24 to 30 months. In addition to the already described similarities in bone physiology, they show good postoperative healing assets and a high indolence after surgery [23]. All animals used in this study were specifically bred for laboratory animal use (Ellegaard Göttingen Minipigs A/S, Denmark). The experimental project with the number $112 / 15$ was approved by the animal welfare Commission of the Office for Health and Consumer Protection, Hamburg (Germany). The experiments were carried out according to the European Communities Council Directive of 24 November 1986 (86/609/EEC) and in accordance with German laws and regulations.

2.2. Surgical Procedure. A perioperative antibiotic prophylaxis was administered for 7 days (Unacid ${ }^{\circledR} 375 \mathrm{mg}$ ). Each animal was injected i.m. with a weight-dependent dose of ketamine $10 \%$, xylazine $2 \%$, and $3 \mathrm{ml}$ of Stresnil ${ }^{\mathrm{TM}}$. Depending on the depth of sedation, $2-4 \mathrm{mg}$ propofol $2 \%$ i.v. was administered. With the inhalation anesthetic, Isoflurane-Baxter $1.5 \%$, the anesthetic was maintained. As volume replacement, Jonosteril ${ }^{\circledR} 1.51$ and HAES-sterile 0.51 were infused during the procedure. To ensure a sufficient analgesia, a vestibular and oral local infiltration of $5 \mathrm{ml}$ of Ultracain ${ }^{\circledR} 4 \%$ with adrenaline $1: 100.000$ was given.

Thereafter a bilateral extraction of the first mandibular molar (M1) and two mandibular premolars (P3 and P4) was conducted, whereby the extraction was carried out atraumatically by piezosurgery (Piezosurgery 3, mectron, Cologne, Germany) with an osteotomy insert, a periotom, extraction levers, and pliers. Six implants were immediately placed in the extraction sockets on each side. The distance in between the implants was above $3 \mathrm{~mm}$ in order to prevent any influences on osseointegration. After extraction, the integrity of the buccal plate was checked with a periodontal probe. An implant bed for implants with regular platform (11.5 mm length, $4.1 \mathrm{~mm}$ diameter, Semados S-Line, BEGO, Bremen, Germany) was prepared according to manufactures guidelines and 6 implants on each side of the jaw were inserted. Implants were covered by a mucosal flap (see Figures 1 and 2).

To check the optimal clinical healing process, a followup examination in general anesthesia was conducted a week and a month after surgery. Wound dehiscences were recorded and if needed restitched after refreshing and cleaning of the edges with chlorhexidingluconate. Each implant loss was documented.

2.3. Animal Sacrifice, Biopsy Harvesting, and Processing. The peri-implant bone level was determined radiographically by CBCT images (SCS MedSeries ${ }^{\circledR}$ Verity H22e, SCS Software Computer Solutions GmbH; Aschaffenburg, Germany) with teeth in place, after extraction/before implantation and after implantation. After a 12 -week healing period, all eight minipigs were sacrificed by intravenous applications of T61 (200 mg 


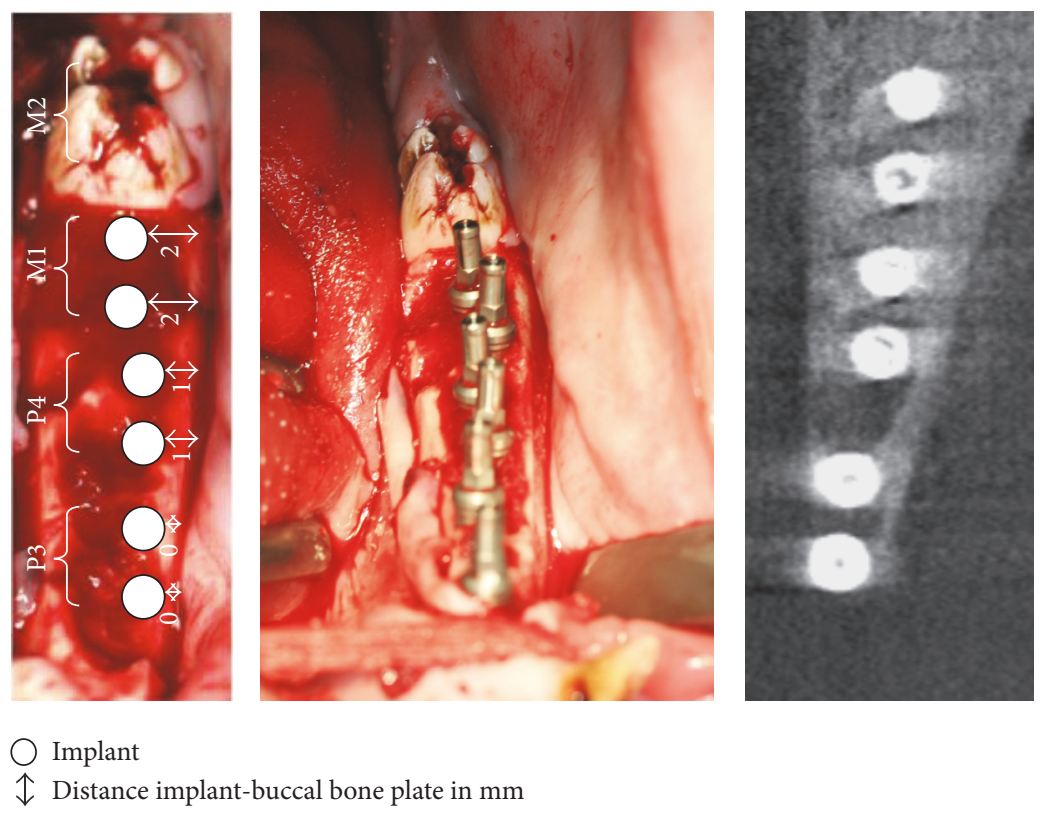

FIGURE 2: Clinical assessment and CBCT after surgery.

embutramide, $50 \mathrm{mg}$ mebezonium, and $5 \mathrm{mg}$ tetracaine per $\mathrm{ml} ; 6 \mathrm{mg} / 50 \mathrm{~kg}$ ) after deep sedation.

After euthanasia, the resection of the relevant sections of the jawbone were implemented with an oscillating bone saw. Standardized CBCT were made by the nonfixed tissue samples and morphometrically analyzed [24-26]. High Resolution CBCT (SCS MedSeries ${ }^{\circledR}$ Verity H22e, SCS Software Computer Solutions GmbH; Aschaffenburg, Germany) recordings were taken of all 8 minipigs. The SCS MedSeries Verity $\mathrm{H} 22 \mathrm{e}$ was used due to specimens shape and size which lead to limitations in correct positioning within a stand-up device. It is usually intended to be used for X-ray computed tomography imaging of anatomies within upper and lower extremities. As the setting and specification of the X-ray tube and the flat panel detector match stand-up CBCTscanners for imaging of the maxillofacial region, the results of measurements and image evaluation can be transferred. For the CBCT scans, the voxel size was set to $200 \mu \mathrm{m}$ in a standard scan protocol with a $193 \times 242 \mathrm{~mm}$ field of view. Tube settings were $90 \mathrm{kV}$ and $6 \mathrm{~mA}$ with $6 \mathrm{~s}$ exposure time. This setting was chosen in order to reflect clinical routine.

The specimens were separated from the surrounding tissue with a diamond band saw (EXAKT Apparatebau, Norderstedt, Germany) perpendicular to the buccal bone surface. Each tissue slice was documented by contact radiography (Faxitron, Tucson, USA). In order to produce the undecalcified histological specimen [27, 28], all samples were dehydrated using an ascending alcohol series (70\%, $80 \%, 96 \%$, and $100 \%$ ethanol), infiltrated with synthetic medium (Technovit 7200 VLC, Heraeus Kulzer, Wehrheim, Germany) and polymerized with blue light. After hardening, the specimens were processed with the cutting-grinding technique (Exakt, Norderstedt, Germany) to a ground section with a thickness of $30 \mu \mathrm{m}$. No specimen was lost due to the procedure. After that, all specimens were stained with toluidine blue. Two independent observers (C. P. and J. M.) measured the thickness of the buccal bone perpendicular to the ninth implant thread and the distance from the implant shoulder to the ninth implant thread in the histologic ground section. Measurements were done with a microscope and defined magnification (50x) using an image analysis system (OsteoMeasure, OsteoMetrics, Decatur, USA) after calibration on test implants.

2.4. Radiologic Measurements in CBCT. Altogether 60 implants could be evaluated in thin sections and CBCT images (for parameters, see Section 2.3). CBCT measurements were conducted in the software OsiriX MD (Version 3.0). Each implant was aligned in the same fashion and implants lengths were calibrated. The histologic measurements from implant shoulder to the ninth thread were transferred to the CBCT measurement software and the buccal bone thickness was measured individually by four different raters (J. S., oral surgeon; M. S., oral and maxillofacial surgeon; J. M., dentist; C. P., oral and maxillofacial surgeon; all are well trained reviewing CBCTs) (see Figure 3). The raters were blinded regarding the histologic measurement results.

2.5. Statistical Evaluation. Mean values and standard deviations were calculated for each parameter per animal. Random intercept models were used; variances and the Interclass Correlation Coefficient (ICC, Interrater Correlation) were calculated. The significance level was defined at $p<0,05$. For assessing the agreement between CBCT and histology, we performed Bland-Altman plots. Mean differences $\pm 2 \times$ standard deviation were computed [29]. All statistical analyses and plots were conducted with R Version 3.3.3 from the RProject for statistical Computing (Vienna, Austria 2017) [30]. 


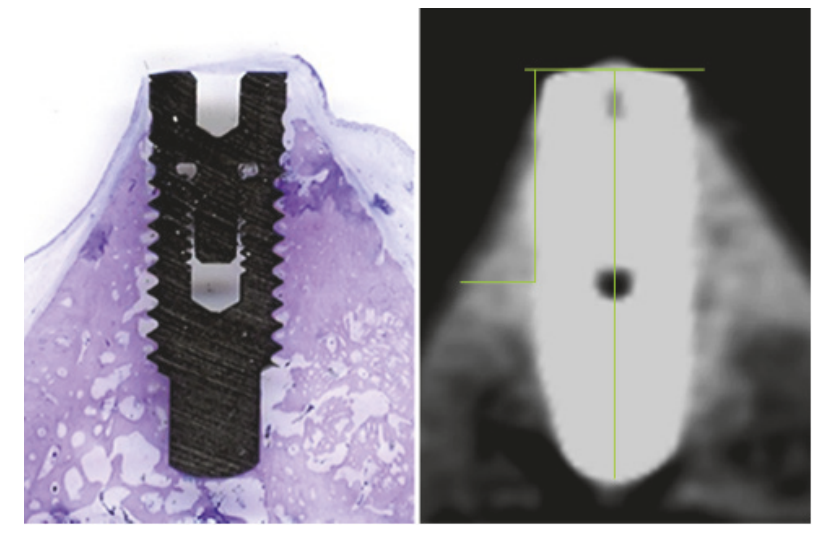

FIGURE 3: CBCT image with measurement lines and corresponding histologic slice specimen.

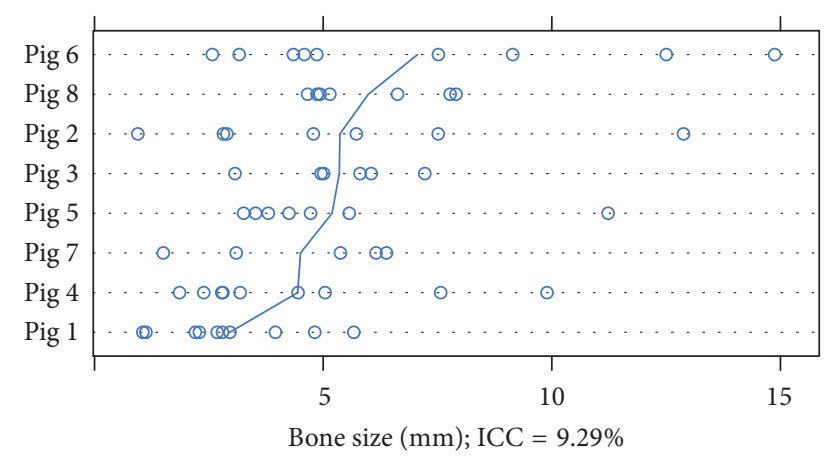

FIGURE 4: Dotplot for single values of bone size ( $\mathrm{mm}$ ) in descending order referring to mean value of the pig in histology. ICC: Intraclass Correlation Coefficient.

\section{Results}

Out of 96 inserted 36 implants had to be excluded from this study because histology and radiologic planes could not be aligned along the long axis of the implant due to the two using standardized protocols. Thus, CBCT images could be oriented in any direction in space, but not the histological sections accordingly. From 60 implants, bone thickness measurements in histology could be compared to bone thickness measurements in CBCT.

3.1. Mean Bone Thickness in Histology. The mean bone thickness per implant measured in histology is pictured in Figure 4. The mean bone thickness was calculated in a random intercept model adjusted for correlated data due to the factor "pig." The bone thickness was defined as fixed effect and the pig ID was the random effect. Controlled by the cluster "pig," the mean of the bone thickness was $5.09 \mathrm{~mm}$ (CI $4.11-6.08 \mathrm{~mm}$ ). The part of variance due to the pig was $9.29 \%$ (CI $0.00-25.14 \%$ ) of total variance (Intraclass Correlation Coefficient (ICC)). The random effect was not significant ( $p$ value 0.02 ; see Figure 5).

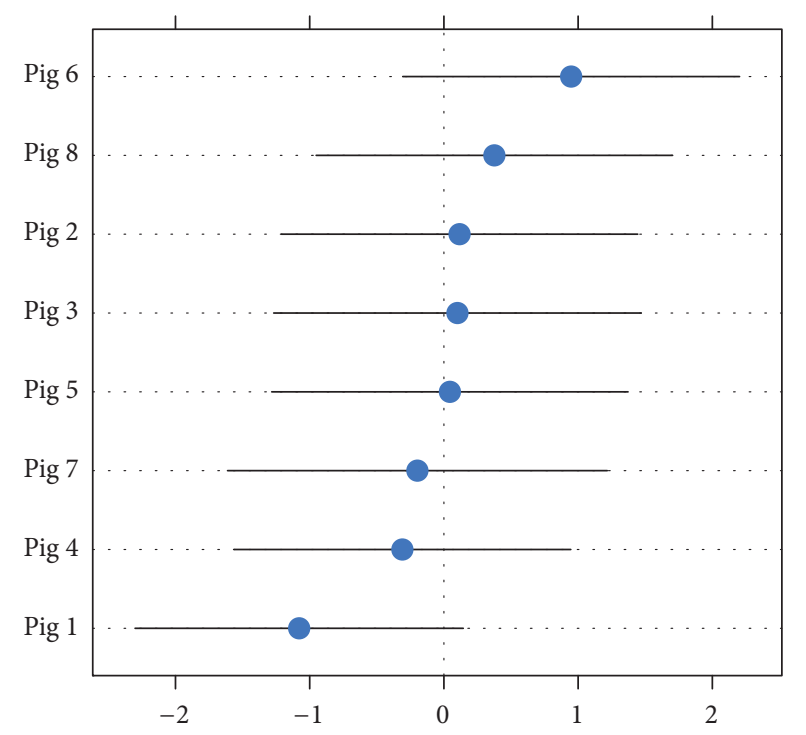

FIGURE 5: Distribution of random effects "pig."

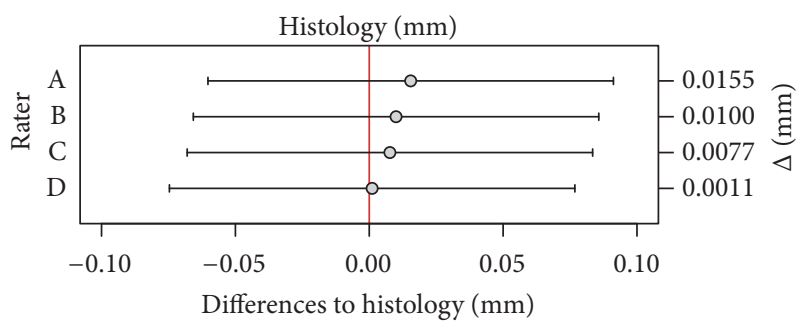

FIGURE 6: Means of bone thickness and confidence intervals measured by raters in CBCT compared to mean of bone thickness in histology.

3.2. Mean Bone Thickness in CBCT. Four random intercept models were calculated for the four raters with bone thickness in CBCT as fixed effect and pig ID as random effect. The mean bone thickness for each rater was as follows: Rater A: $5.10 \mathrm{~mm}$ (CI 4.12-6.10 mm); Rater B: $5.09 \mathrm{~mm}$ (CI 4.11-6.10 mm); Rater C: 5.09 mm (CI 4.09-6.12 mm); Rater D: 5.09 mm (CI 4.11-6.08 mm) (see Figure 6).

3.3. Differences of Bone Thickness in Histology and CBCT. The deviations of mean bone thickness measured by the raters in CBCT from the mean bone thickness in histology were marginal. All raters measured slightly more bone in CBCT than in histology (see Figures 6, 7, and 8). Averaged quarter millimeter more or less was measured compared to histology. The variance of pigs is compared to the variance of implants, not significant. The part of the variance due to rater was $1.35 \%$ of total variance (calculated without part of variance due to pig). The confidence interval (CI) was $0.00-3.01 \%$ of total variance. The random effect was not significant ( $p$ value 1.000). Therefore the Intraclass Correlation Coefficient (ICC, Interrater Correlation) was 98.65\% (CI 100.00-96.99\%). The Bland-Altman plot shows the range in bone thickness, plotted against the differences comparing histology and CBCT measurements for each rater (see Figure 8). 


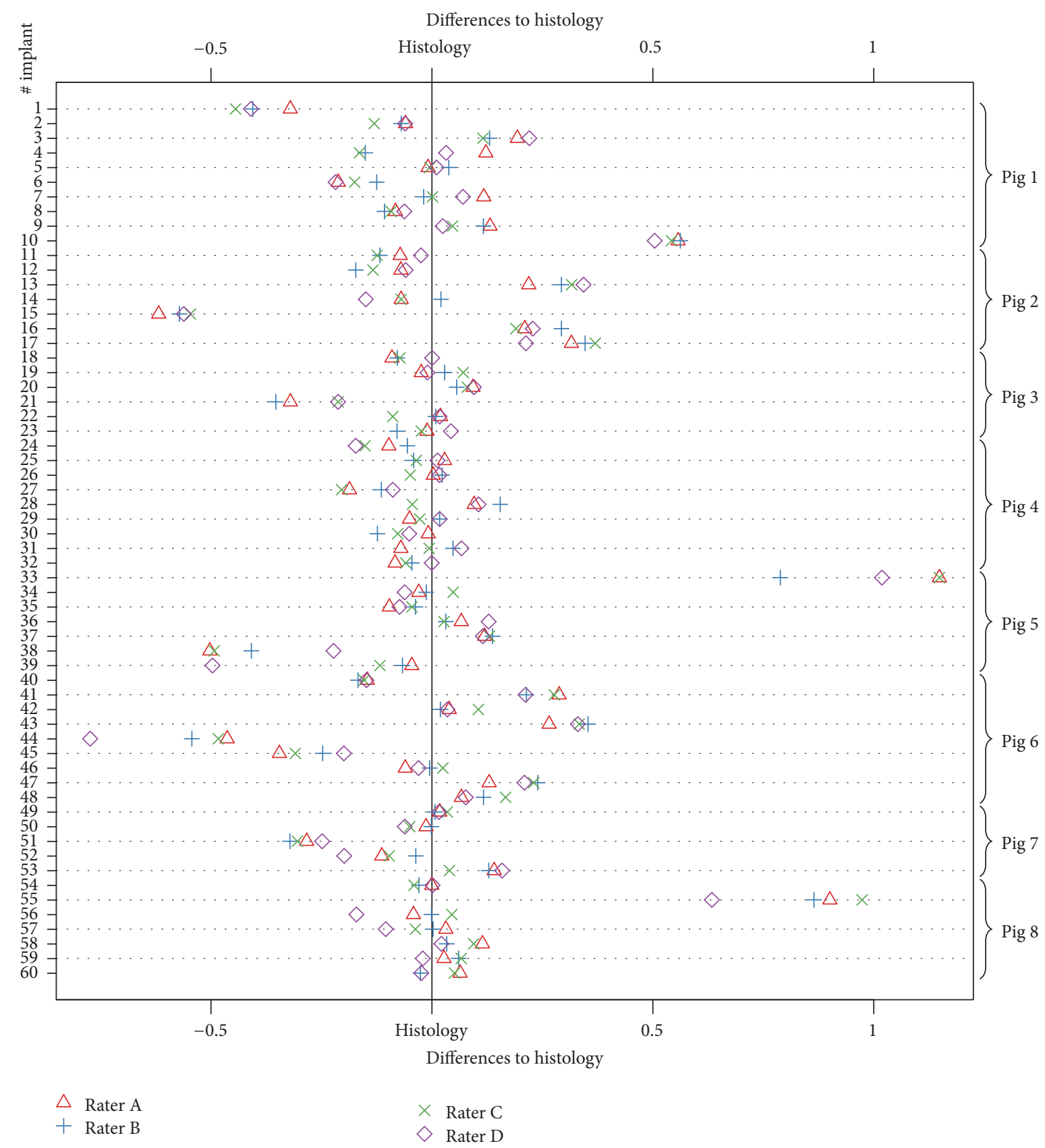

FIgURE 7: Differences of bone measurements in CBCT and histology (in mm).

3.4. Correlation between Measurement Accuracy and Bone Thickness. To test the measurement accuracy in small bone sizes, the data set was grouped in " $<3 \mathrm{~mm}$ " versus " $\geq 3 \mathrm{~mm}$ " and " $<2 \mathrm{~mm}$ " versus " $\geq 2 \mathrm{~mm}$." A random intercept model was calculated while controlling for cluster effect "pig” and "rater." The means of the groups $\geq 3 \mathrm{~mm}(0.033 \mathrm{~mm})$ and $\geq 2 \mathrm{~mm}(0.019 \mathrm{~mm})$ were slightly overestimated compared to histology, whereas the means of the groups $<3 \mathrm{~mm}$ $(-0.071 \mathrm{~mm})$ and $<2 \mathrm{~mm}(-0.099 \mathrm{~mm})$ were slightly underestimated. The mean difference of each measurement compared to histology was in group $<3 \mathrm{~mm} 0.152 \mathrm{~mm}$, in group $\geq 3 \mathrm{~mm} 0.287 \mathrm{~mm}$, in group $<2 \mathrm{~mm} 0.170 \mathrm{~mm}$, and in group $\geq 2 \mathrm{~mm} 0.271 \mathrm{~mm}$. The ICC was in group $<3 \mathrm{~mm} 99.32 \%$ (CI
$100.00-91.42 \%$ ), in group $\geq 3 \mathrm{~mm} 99.79 \%$ (CI 100-97.13\%), in group $<2 \mathrm{~mm} 97.47 \%$ (CI 100-83.45\%), and in group $\geq 2 \mathrm{~mm}$ $100 \%$ (CI 100.00-97.35\%).

An increase of one millimeter in bone thickness in CBCT leads to an additional average increase of measurement of $0.018 \mathrm{~mm}$ (CI $0.0006-0.030 \mathrm{~mm})$. This effect is significant $(p$ value 0.003) (see Figure 9).

\section{Discussion}

In this study, dental implants were inserted in pig jaws immediately after tooth extraction. Cone-beam computed tomographies were taken directly after scarification of the 

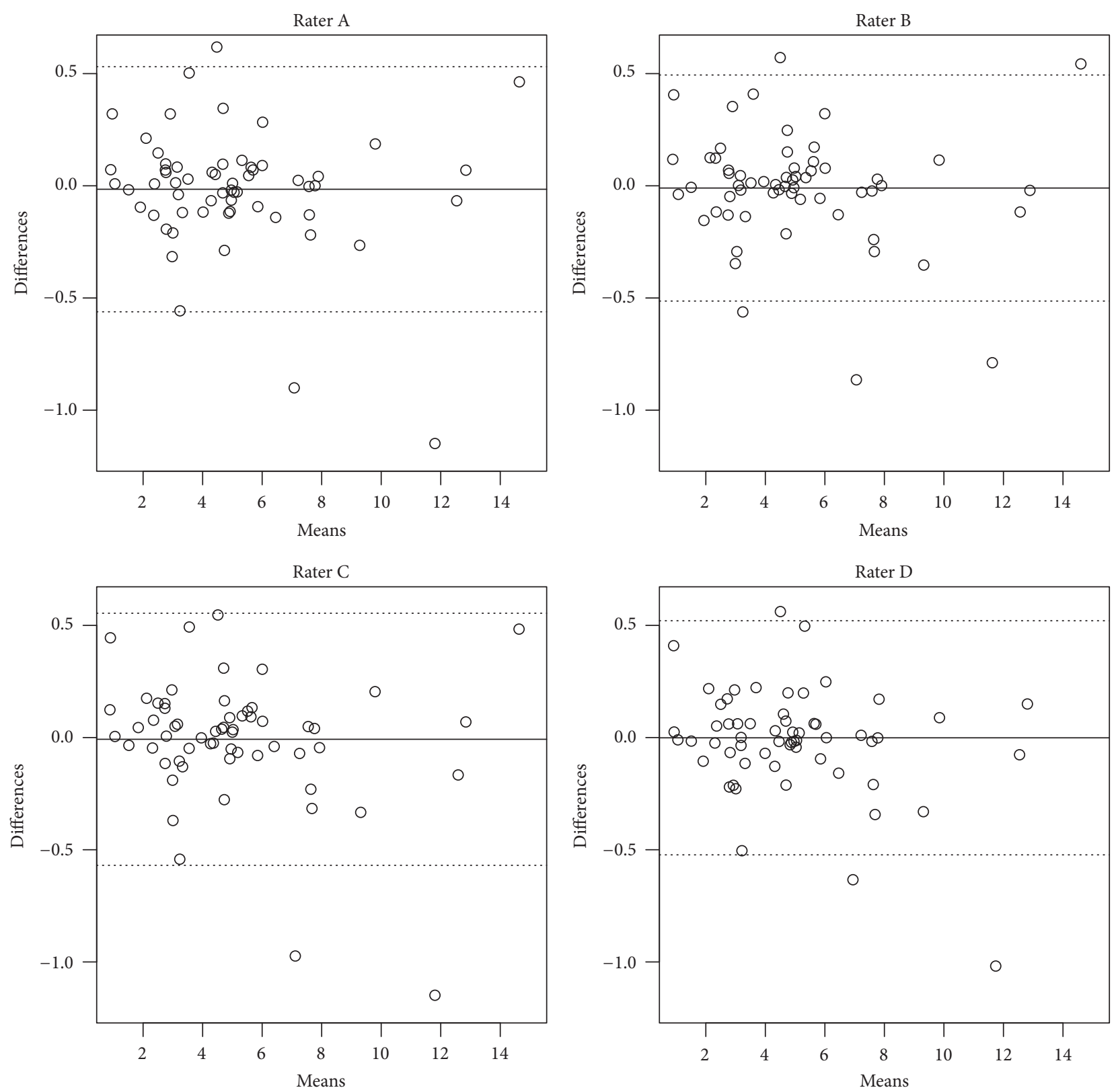

FIGURE 8: Bland-Altman plot: histology plotted against raters A-D.

animals 6 months after surgery and the implants with surrounding bone tissue were processed histologically. The bone thickness directly around the implants was measured and compared correspondingly in histological sections and in CBCT datasets.

Bone thickness is crucial for a good initial implant stability and initial implant stability important for implant success [31]. Furthermore alveolar bone loss around implants over time is an important index for peri-implantitis and can lead to implant loss. For identification, the need of accurate measurement in CBCT datasets as a diagnostic tool is obvious. Most studies compared clinical assessment with radiologic measurement using different acquisition parameters and measurement tools [32-36]. CBCT was evaluated in staging periodontitis, determining alveolar bone defects and bone assessment around dental implants [32, 37-42]. Most of these studies stated, that CBCT images are adequate for the assessment of alveolar bone [21, 31, 35, 43, 44]. Nevertheless there are only a small number of studies comparing the CBCT measurements to histological measurements such as the present study. Ritter et al. placed 26 dental implants in dog jaws with chronic type vestibular defects and correlated CBCT measurements with histomorphometry of the vestibular bone level, oral bone thickness, and implant length. They found that 3D CBCT provides usable information about bone in all dimensions around implants with varying accuracy [43]. Wang et al. examined artificial defects in foxhounds after guided bone regeneration and implantation. They measured 


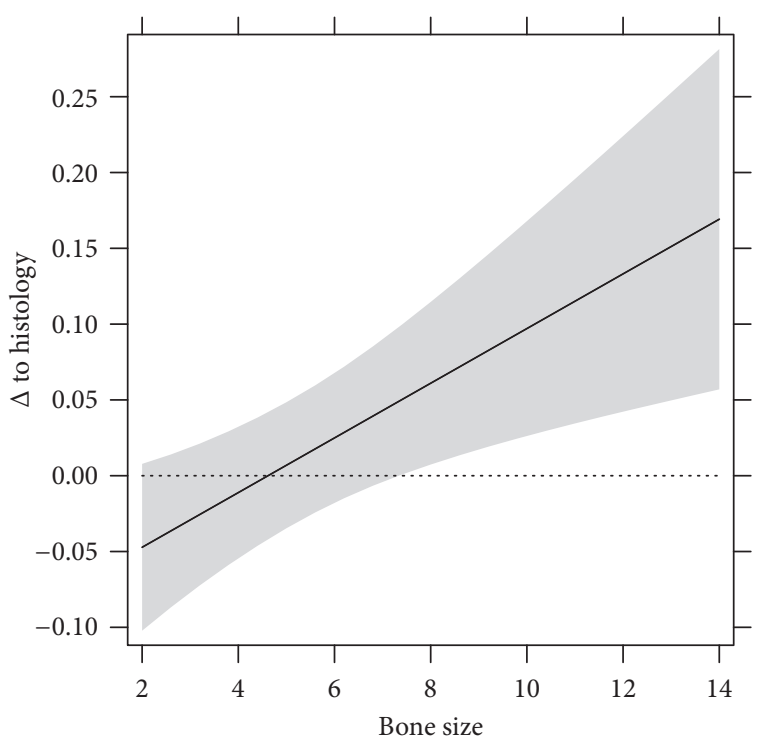

Figure 9: Difference of CBCT measurements to measurements in histology and influence of bone thickness (in $\mathrm{mm}$ ) on average deviation.

the bone thickness in a orobuccal dimension of 41 implants at different levels and thus evaluated the integration of different bone augmentation materials. Thereafter, peri-implant bone thickness could be measured at an accuracy of half a millimeter. The assessment of the existence and integration of bone augmentation material was partially possible [31]. In the present study, altogether 60 implants were evaluated by CBCT in correspondence to its histology. All raters measured slightly more bone in CBCT than in histology. By average, a quarter millimeter more or less was measured compared to histology. As voxel size was $0.2 \mathrm{~mm}$ in the present study, these findings are within expected technical limitations due to the given resolution and thus could be interpreted as technical or imaging artifacts.

Ritter et al. found an overestimation of about $+0.3 \mathrm{~mm}$ $( \pm 0.04 \mathrm{~mm})$ on average when comparing CBCT and histology. They stated that the direction of the implant artifacts is diagonal to the implant axis, which is why bone seems wider [43] as in reality. By forming groups (either $\geq 3 \mathrm{~mm} /<3 \mathrm{~mm}$ or $\geq 2 \mathrm{~mm} /<2 \mathrm{~mm}$ ) in this study, smaller bone sizes are tending to be underestimated compared to wider bone sizes and higher bone sizes which were overestimated. An increase of one millimeter in bone thickness in CBCT increases the average measurement to additional $0.02 \mathrm{~mm}$ (CI 0.01-0.03 mm). This effect is significant ( $p$ value 0.003 ). González-Martín et al. presented a similar finding. For each millimeter of increase of bone thickness, the odds of radiographic identification increased by $30.6(p<0.001)$ [34]. Razavi et al. stated that above $1 \mathrm{~mm}$ of bone thickness, the accuracy of CBCT images increased substantially [45]. Dos Santos Corpas et al. found that the used CBCT deviates $1.20 \mathrm{~mm}$ from the histology when examining bone defects [38]. Fienitz et al. showed that CBCT is not accurate in sites of bone width lower than $0.5 \mathrm{~mm}$ [39]. The Interrater Correlation in the present and other studies was very high (98.65\% (CI 100.00-96.99\%)), indicating that CBCT is a reliable method for measuring bone structures [31, 35].

But, limitations in the present study are the use of CBCT scans obtained from isolated blocks of dead tissues and not from alive minipigs. Thus, CBCT datasets are representative of a best case scenario as CBCT has been shown at least for thin bone areas to be less accurate in living patients and different compared to what has been found in cadaver studies.

\section{Conclusion}

In this study there was no significant difference between CBCT measurement and corresponding histologic sections, indicating that $\mathrm{CBCT}$ is a valuable diagnostic tool for determining bone thickness in direct surrounding of dental implants without relevant artifacts. Thus, CBCT imaging is a reliable diagnostic tool to determine peri-implant bone loss and therefore estimate the risk of implant loss and the prognosis of implant survival. Another important clinical field could be the diagnosis of bone invasion from, for example, oral squamous cell carcinoma during tumor followup and staging without interference of CT specific metal artifacts.

\section{Conflicts of Interest}

The implants used in this study were kindly provided by BEGO (Bremen, Germany). The authors declare no other conflicts of interest.

\section{Authors' Contributions}

Alexander Gröbe and Jan Semmusch contributed equally to this work and should be considered as joint first authors.

\section{References}

[1] P. Mozzo, C. Procacci, A. Tacconi, P. Tinazzi Martini, and I. A. Bergamo Andreis, "A new volumetric CT machine for dental imaging based on the cone-beam technique: preliminary results," European Radiology, vol. 8, no. 9, pp. 1558-1564, 1998.

[2] D. Schulze, M. Heiland, H. Thurmann, and G. Adam, "Research: Radiation exposure during midfacial imaging using 4- and 16slice computed tomography, cone beam computed tomography systems and conventional radiography," Dentomaxillofacial Radiology, vol. 33, no. 2, pp. 83-86, 2004.

[3] S. K. Koskinen, V. V. Haapamäki, J. Salo et al., "CT arthrography of the wrist using a novel, mobile, dedicated extremity conebeam CT (CBCT)," Skeletal Radiology, vol. 42, no. 5, pp. 649657, 2013.

[4] M. T. Truong, A. E. Hirsch, N. Kovalchuk et al., "Conebeam computed tomography image guided therapy to evaluate lumpectomy cavity variation before and during breast radiotherapy," Journal of Applied Clinical Medical Physics, vol. 14, no. 2, pp. 209-219, 2013.

[5] S. Veldhoen, M. Schöllchen, H. Hanken et al., "Performance of cone-beam computed tomography and multidetector computed tomography in diagnostic imaging of the midface: A comparative study on Phantom and cadaver head scans," European Radiology, vol. 27, no. 2, pp. 790-800, 2016. 
[6] A. W. F. Hendrikx, T. Maal, F. Dieleman, E. M. Van Cann, and M. A. W. Merkx, "Cone-beam CT in the assessment of mandibular invasion by oral squamous cell carcinoma: results of the preliminary study," International Journal of Oral and Maxillofacial Surgery, vol. 39, no. 5, pp. 436-439, 2010.

[7] J. B. Ludlow and M. Ivanovic, "Comparative dosimetry of dental CBCT devices and 64-slice CT for oral and maxillofacial radiology," Oral Surgery, Oral Medicine, Oral Pathology, Oral Radiology and Endodontology, vol. 106, no. 1, pp. 930-938, 2008.

[8] M. Loubele, R. Bogaerts, E. van Dijck et al., "Comparison between effective radiation dose of CBCT and MSCT scanners for dentomaxillofacial applications," European Journal of Radiology, vol. 71, no. 3, pp. 461-468, 2009.

[9] C. Holberg, S. Steinhäuser, P. Geis, and I. Rudzki-Janson, "Cone-beam computed tomography in orthodontics: benefits and limitations," Journal of Orofacial Orthopedics, vol. 66, no. 6, pp. 434-444, 2005.

[10] E. Hofmann, M. Schmid, M. Sedlmair, R. Banckwitz, U. Hirschfelder, and M. Lell, "Comparative study of image quality and radiation dose of cone beam and low-dose multislice computed tomography—an in-vitro investigation," Clinical Oral Investigations, vol. 18, no. 1, pp. 301-311, 2014.

[11] A. Suomalainen, T. Kiljunen, Y. Käser, J. Peltola, and M. Kortesniemi, "Dosimetry and image quality of four dental cone beam computed tomography scanners compared with multislice computed tomography scanners," Dentomaxillofacial Radiology, vol. 38, no. 6, pp. 367-378, 2009.

[12] D. Schulze, M. Heiland, H. Thurmann, and G. Adam, "Radiation exposure during midfacial imaging using 4- and 16slice computed tomography, cone beam computed tomography systems and conventional radiography," Dentomaxillofacial Radiology, vol. 33, no. 2, pp. 83-86, 2004.

[13] R. Guijarro-Martínez and G. R. J. Swennen, "Cone-beam computerized tomography imaging and analysis of the upper airway: a systematic review of the literature," International Journal of Oral and Maxillofacial Surgery, vol. 40, no. 11, pp. 1227-1237, 2011.

[14] R. E. Friedrich, M. Bruhn, and C. Lohse, "Cone-beam computed tomography of the orbit and optic canal volumes," Journal of Cranio-Maxillofacial Surgery, vol. 44, no. 9, pp. 1342-1349, 2016.

[15] A. Insua, A. Monje, H.-L. Chan, N. Zimmo, L. Shaikh, and H.-L. Wang, "Accuracy of Schneiderian membrane thickness: a cone-beam computed tomography analysis with histological validation," Clinical Oral Implants Research, 2016.

[16] S. Kanagasingam, C. X. Lim, C. P. Yong, F. Mannocci, and S. Patel, "Diagnostic accuracy of periapical radiography and cone beam computed tomography in detecting apical periodontitis using histopathological findings as a reference standard," International Endodontic Journal, 2016.

[17] J. Wikner, H. Hanken, C. Eulenburg et al., "Linear accuracy and reliability of volume data sets acquired by two CBCT-devices and an MSCT using virtual models: a comparative in-vitro study," Acta Odontologica Scandinavica, vol. 74, no. 1, pp. 51-59, 2016.

[18] F. G. Draenert, E. Coppenrath, P. Herzog, S. Müller, and U. G. Mueller-Lisse, "Beam hardening artefacts occur in dental implant scans with the NewTom ${ }^{\circledR}$ cone beam CT but not with the dental 4-row multidetector CT," Dentomaxillofacial Radiology, vol. 36, no. 4, pp. 198-203, 2007.

[19] R. Schulze, U. Heil, D. Groß et al., "Artefacts in CBCT: a review," Dentomaxillofacial Radiology, vol. 40, no. 5, pp. 265-273, 2011.
[20] M. Sancho-Puchades, C. H. F. Hämmerle, and G. I. Benic, "In vitro assessment of artifacts induced by titanium, titaniumzirconium and zirconium dioxide implants in cone-beam computed tomography," Clinical Oral Implants Research, vol. 26, no. 10, pp. 1222-1228, 2015.

[21] L. N. Shiratori, J. Marotti, J. Yamanouchi, I. Chilvarquer, I. Contin, and P. Tortamano-Neto, "Measurement of buccal bone volume of dental implants by means of cone-beam computed tomography," Clinical Oral Implants Research, vol. 23, no. 7, pp. 797-804, 2012.

[22] H. H. Sambraus, "Endangered domestic animal breeds in German-speaking countries. IV. Swine and goats," Tierarztl Prax, vol. 15, no. 2, pp. 117-122, 1987.

[23] R. Beglinger, “The Goettingen miniature swine as an experimental animal. 1. Review of literature, breeding and handling cardiovascular parameters," Research in Experimental Medicine, vol. 165, no. 3, pp. 251-263, 1975.

[24] I. Kim, K.-S. Paik, and S.-P. Lee, "Quantitative evaluation of the accuracy of micro-computed tomography in tooth measurement," Clinical Anatomy, vol. 20, no. 1, pp. 27-34, 2007.

[25] C. H. Park, Z. R. Abramson, M. Taba Jr. et al., "Threedimensional micro-computed tomographic imaging of alveolar bone in experimental bone loss or repair," Journal of Periodontology, vol. 78, no. 2, pp. 273-281, 2007.

[26] T. N. R. Clementino-Luedemann and K.-H. Kunzelmann, "Mineral concentration of natural human teeth by a commercial micro-CT," Dental Materials Journal, vol. 25, no. 1, pp. 113-119, 2006.

[27] K. Donath and G. Breuner, "A method for the study of undecalcified bones and teeth with attached soft tissues: the Sage-Schliff (sawing and grinding) technique," Journal of Oral Pathology, vol. 11, no. 4, pp. 318-326, 1982.

[28] M. Hahn, M. Vogel, and G. Delling, "Undecalcified preparation of bone tissue: report of technical experience and development of new methods," Virchows Archiv A Pathological Anatomy and Histopathology, vol. 418, no. 1, pp. 1-7, 1991.

[29] J. M. Bland and D. G. Altman, "Statistical methods for assessing agreement between two methods of clinical measurement," The Lancet, vol. 1, no. 8476, pp. 307-310, 1986.

[30] R Core Team, "A Language and Environment for Statistical Computing," R Foundation for Statistical Computing, Vienna, Austria, 2017.

[31] D. Wang, A. Künzel, V. Golubovic et al., "Accuracy of periimplant bone thickness and validity of assessing bone augmentation material using cone beam computed tomography," Clinical Oral Investigations, vol. 17, no. 6, pp. 1601-1609, 2013.

[32] Y. Guo, Z. Ge, R. Ma, J. Hou, and G. Li, "A six-site method for the evaluation of periodontal bone loss in cone-beam CT images," Dentomaxillofacial Radiology, vol. 45, no. 1, p. 20150265, 2016.

[33] C. T. Castro-Ruiz, J. Noriega, and M. E. Guerrero, "Validity of ridge mapping and cone beam computed tomography in dental implant therapy," Journal of Indian Society of Periodontology, vol. 19, no. 3, pp. 290-293, 2015.

[34] O. González-Martín, C. Oteo, R. Ortega, J. Alandez, M. Sanz, and M. Veltri, "Evaluation of peri-implant buccal bone by computed tomography: An experimental study," Clinical Oral Implants Research, vol. 27, no. 8, pp. 950-955, 2016.

[35] V. C. Cook, A. M. Timock, J. J. Crowe, M. Wang, and D. A. Covell, "Accuracy of alveolar bone measurements from cone beam computed tomography acquired using varying settings," Orthodontics and Craniofacial Research, vol. 18, no. 1, pp. 127136, 2015. 
[36] R. Patcas, L. Müller, O. Ullrich, and T. Peltomäki, "Accuracy of cone-beam computed tomography at different resolutions assessed on the bony covering of the mandibular anterior teeth," American Journal of Orthodontics and Dentofacial Orthopedics, vol. 141, no. 1, pp. 41-50, 2012.

[37] K. de Faria Vasconcelos, K. M. Evangelista, C. D. Rodrigues, C. Estrela, T. O. de Sousa, and M. A. G. Silva, "Detection of periodontal bone loss using cone beam CT and intraoral radiography," Dentomaxillofacial Radiology, vol. 41, no. 1, pp. 64-69, 2012.

[38] L. Dos Santos Corpas, R. Jacobs, M. Quirynen, Y. Huang, I. Naert, and J. Duyck, "Peri-implant bone tissue assessment by comparing the outcome of intra-oral radiograph and cone beam computed tomography analyses to the histological standard," Clinical Oral Implants Research, vol. 22, no. 5, pp. 492-499, 2011.

[39] T. Fienitz, F. Schwarz, L. Ritter, T. Dreiseidler, J. Becker, and D. Rothamel, "Accuracy of cone beam computed tomography in assessing peri-implant bone defect regeneration: a histologically controlled study in dogs," Clinical Oral Implants Research, vol. 23, no. 7, pp. 882-887, 2012.

[40] Y. Miyamoto and T. Obama, "Dental cone beam computed tomography analyses of postoperative labial bone thickness in maxillary anterior implants: comparing immediate and delayed implant placement," International Journal of Periodontics and Restorative Dentistry, vol. 31, no. 3, pp. 215-225, 2011.

[41] R. E. Jung, G. I. Benic, D. Scherrer, and C. H. F. Hämmerle, "Cone beam computed tomography evaluation of regenerated buccal bone 5 years after simultaneous implant placement and guided bone regeneration procedures-a randomized, controlled clinical trial," Clinical Oral Implants Research, vol. 26, no. 1, pp. 28-34, 2015.

[42] E. A. Lee, O. Gonzalez-Martin, and J. Fiorellini, "Lingualized flapless implant placement into fresh extraction sockets preserves buccal alveolar bone: a cone beam computed tomography study," The International Journal of Periodontics \& Restorative Dentistry, vol. 34, no. 1, pp. 61-68, 2014.

[43] L. Ritter, M. C. Elger, D. Rothamel et al., "Accuracy of periimplant bone evaluation using cone beam CT, digital intra-oral radiographs and histology," Dentomaxillofacial Radiology, vol. 43, no. 6, Article ID 20130088, 2014.

[44] A. M. Timock, V. Cook, T. McDonald et al., "Accuracy and reliability of buccal bone height and thickness measurements from cone-beam computed tomography imaging," American Journal of Orthodontics and Dentofacial Orthopedics, vol. 140, no. 5, pp. 734-744, 2011.

[45] T. Razavi, R. M. Palmer, J. Davies, R. Wilson, and P. J. Palmer, "Accuracy of measuring the cortical bone thickness adjacent to dental implants using cone beam computed tomography," Clinical Oral Implants Research, vol. 21, no. 7, pp. 718-725, 2010. 


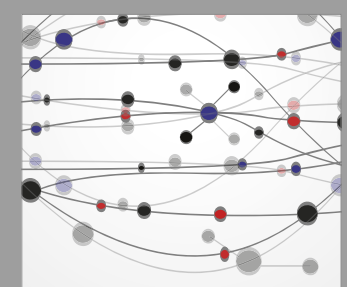

The Scientific World Journal
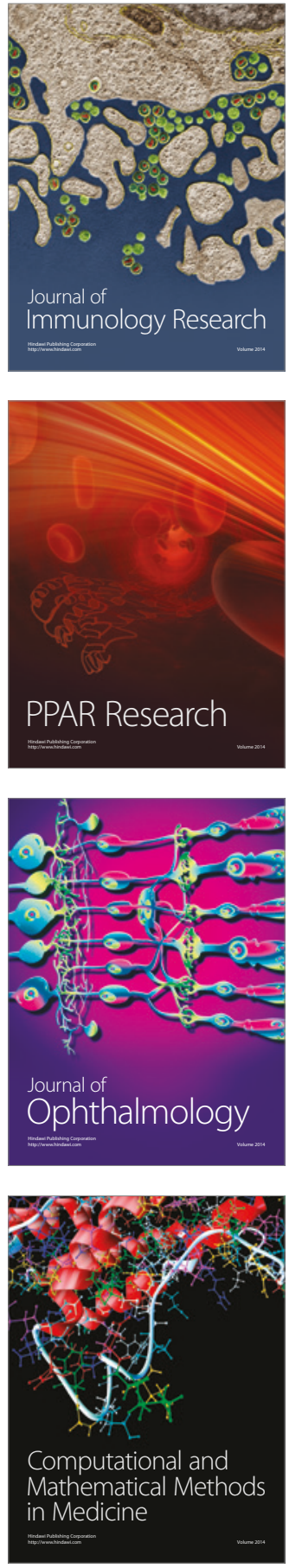

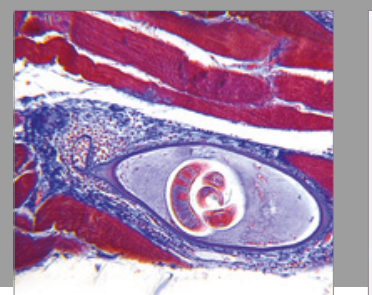

Gastroenterology Research and Practice
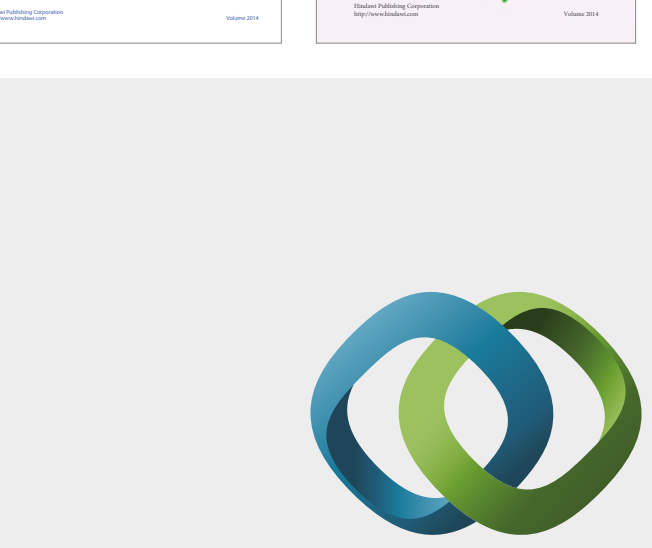

\section{Hindawi}

Submit your manuscripts at

https://www.hindawi.com
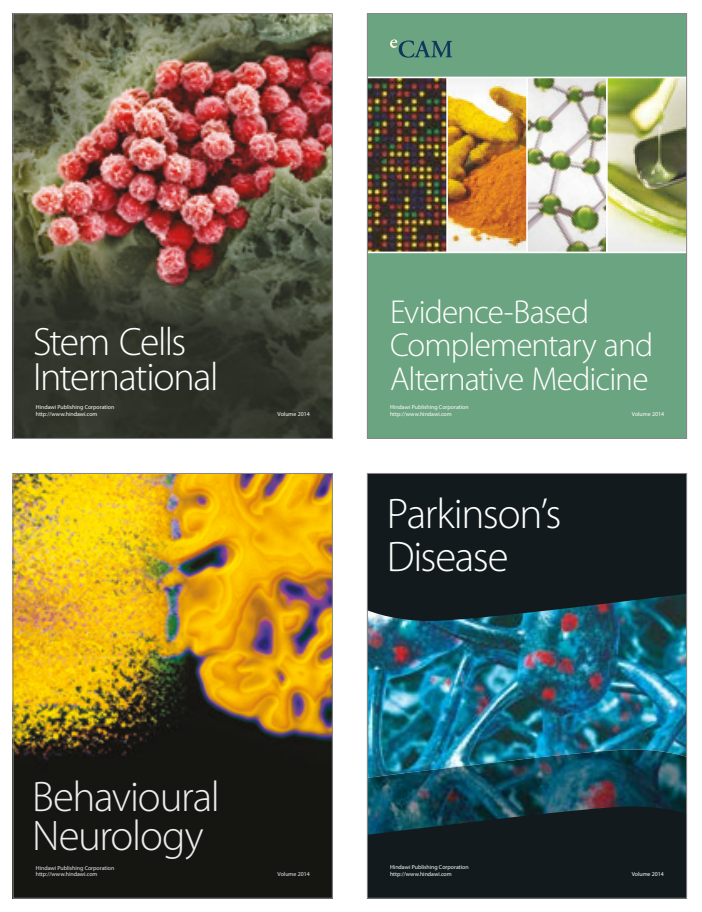
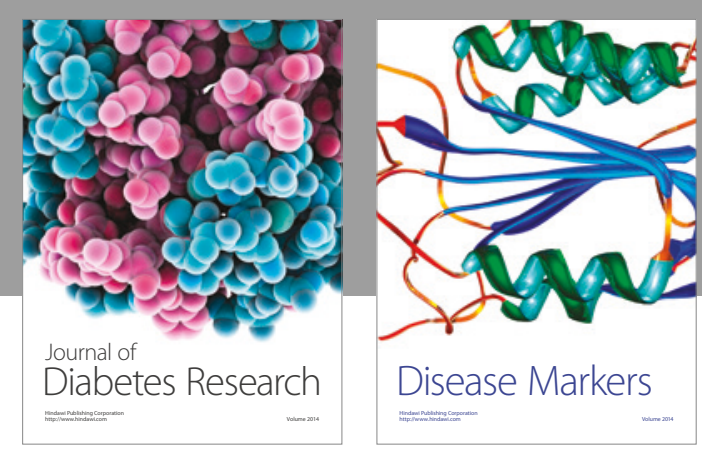

Disease Markers
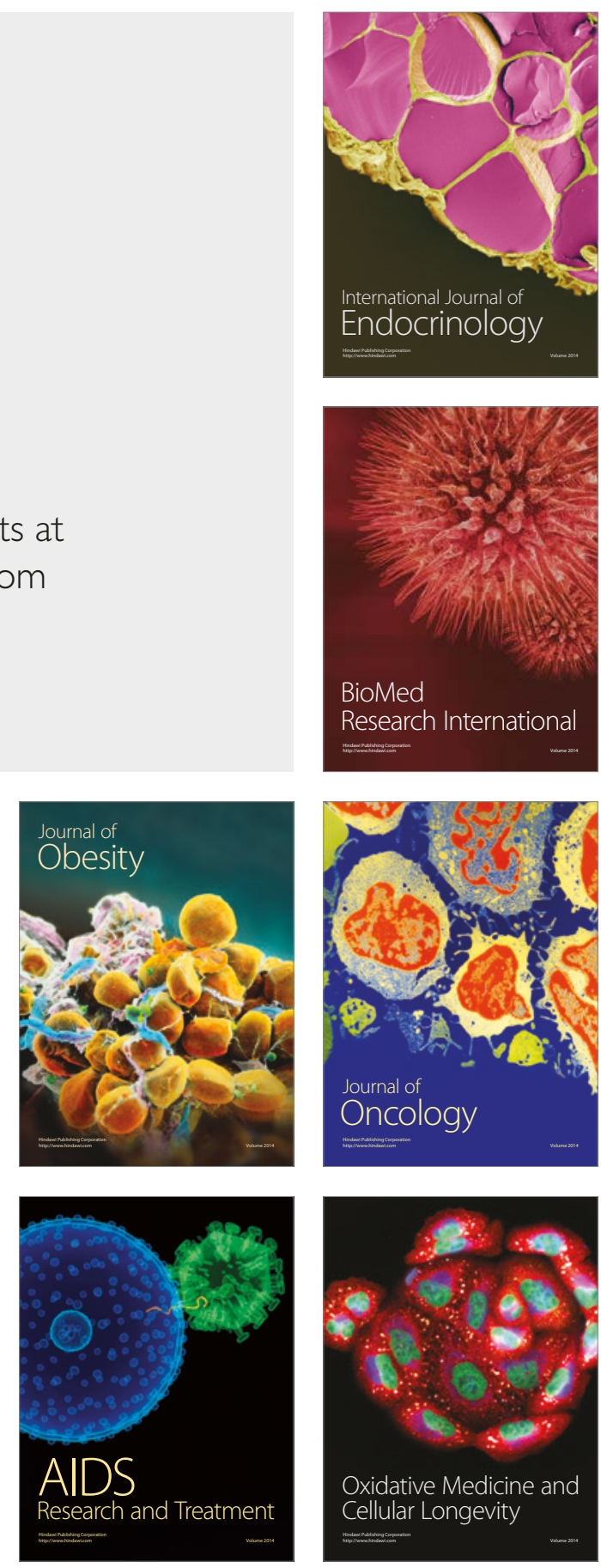